\title{
SUMS AND PRODUCTS OF ORDERED SYSTEMS
}

\author{
PHILIP W. CARRUTH
}

1. Introduction. In this paper we state necessary and sufficient conditions for an ordered sum of ordered systems to be a lattice. Other results concerning ordered sums, similar to those given in $[3, \text { p. 39 }]^{\mathbf{1}}$ concerning the ordinal power of an ordered system, are obtained. Also a number of analogous results relating to ordered products of ordered systems are given.

We shall use some of the notation and definitions of [3]. For the sake of convenience we list here some of those definitions and symbols that will be employed. By an ordered system is meant a nonempty set $R$ of elements in which a reflexive binary relation $r \geqq r^{\prime}$ is defined. Unless otherwise specified, an italic capital letter always will denote an ordered system in the sequel. A subsystem $T$ of $R$ is a subset of elements of $R$ with the order relation in $T$ imposed by that in $R$.

The expressions and symbols maximal element, greatest element, ascending chain condition, isomorphic, $>$, and so on, will have their usual meanings (see [2], for example). The symbols $\vee$ and $\wedge$ will be used in denoting least upper bound (l.u.b.) and greatest lower bound (g.l.b.) respectively. The symbols 0 and $I$ will denote the bounds of bounded ordered systems. The term number will mean partially ordered set. The symbol $S>R$ will mean that $R$ is isomorphic to a subsystem of $S$.

If for each element $r$ in $R, S_{r}$ is an ordered system, the ordered sum over $R$ of the systems $S_{r}$ (denoted by $\sum_{R} S_{r}$ ) is the system $P$ where the elements of $P$ are the ordered pairs $(r, s)$ with $r$ in $R$ and $s$ in $S_{r}$, and $(r, s) \geqq\left(r^{\prime}, s^{\prime}\right)$ means that $r>r^{\prime}$ or else $r=r^{\prime}$ and $s \geqq s^{\prime}$. If all $S_{r}=S$, we write $R \circ S$ for $\sum_{R} S_{r}$. The ordered product over $R$ of the $S_{r}$ (denoted by $\prod_{R} S_{r}$ ) is the system $P$ where the elements of $P$ are the functions $f$ defined on $R$ such that $f(r) \in S_{r}$, while $f \geqq f^{\prime}$ means that if $f(r) \neq f^{\prime}(r)$, there exists $r^{\prime} \geqq r$ such that $f\left(r^{\prime}\right)>f^{\prime}\left(r^{\prime}\right)$.

We list several results in [3] that are used in proofs in this paper.

$[3,2.2]$ states that $\sum_{R} S_{r}>R$ and $\sum_{R} S_{r}>S_{t}$ for every element $t$ in $R$.

$[3,2.4]$ says that $\sum_{R} S_{r}$ is a number if and only if $R$ and all $S_{r}$ are numbers.

$[3,3.9]$ states that $\prod_{R} S_{r}>S_{t}$ for every element $t$ in $R$.

Presented to the Society, April 28, 1951; received by the editors January 6, 1951.

1 Numbers in brackets refer to the bibliography at the end of the paper. 
Let $R_{1}$ be the subsystem of $R$ consisting of all $r$ such that $S_{r}$ is not a cardinal. In part, $[3,3.10]$ states that if all $S_{r}$ are numbers, then $\prod_{R} S_{r}>R_{\mathbf{1}}$. [3, 4.13] states that $\prod_{R} S_{r}$ is a number if and only if (1) all $S_{r}$ and $R_{1}$ are numbers, (2) $R_{1}$ satisfies the ascending chain condition, and (3) if $r^{\prime \prime} \geqq r^{\prime} \geqq r$ in $R$, if $r^{\prime \prime}$ and $r^{\prime} \in R_{1}$, and $S_{r}$ contains more than one element, then $r^{\prime \prime} \geqq r$.

\section{Ordered sums.}

THEOREM 1. $\sum_{R} S_{r}$ is a lattice [complete lattice] if and only if the following conditions are satisfied.

(1) $R$ is a lattice [complete lattice], and all $S_{r}$ are numbers.

(2) Let $P$ be any two element subsystem [any subsystem] of $R$, let $t$ be the l.u.b. of $P$, and let $x$ be the g.l.b. of $P$. If $t \notin P$, then $S_{t}$ contains 0 ; if $x \notin P$, then $S_{x}$ contains $I$.

(3) Let $r$ be any element of $R$, and let $Q$ be any two element subsystem [any subsystem] of $S_{r}$. If there is no l.u.b. of $Q$, then there is no upper bound of $Q, r$ has a least proper successor, say $w$, and $S_{w}$ contains 0 . If there is no g.l.b. of $Q$, then there is no lower bound of $Q, r$ has a greatest proper predecessor, say $y$, and $S_{y}$ contains $I$.

Proof. Assume that $\sum_{R} S_{r}$ is a lattice [complete lattice]. Then it is a number, and $[3,2.4]$ implies that $R$ and all $S_{r}$ are numbers. Let $P$ be a two element subsystem [any subsystem] of $R$. Suppose that there is no greatest element of $P$. Let $s_{p}$, for each element $p$ in $P$, be a fixed element of $S_{p}$. Then $\bigvee_{p \in P}\left(p, s_{p}\right)=($ l.u.b. of $P, 0)$. By a dual argument it is seen that conditions (1) and (2) are satisfied. Let $r$ be any element of $R$, and let $Q$ be any two element subsystem [any subsystem] of $S_{r}$. Suppose that there is no l.u.b. of $Q$. Then $V_{q \in Q}(r, q)=(w, 0)$, where $w$ must be the least proper successor of $r$. Again a dual argument shows that (3) is satisfied.

Now assume that conditions (1), (2), and (3) hold. (1) and [3, 2.4] together imply that $\sum_{R} S_{r}$ is a number.

Let $T$ be any two element subsystem [any subsystem] of $\sum_{R} S_{r}$. Let $P$ be the subsystem of $R$ of all $p$ such that there is an element $(p, s)$ in $T$. If there is a greatest element $u$ of $P$, let $Q$ be the subsystem of $S_{u}$ of all elements $q$ such that $(u, q) \in T$. Then the l.u.b. of $T$ is $(u, 1 . u . b$. of $Q)$ if $Q$ has a l.u.b. If $Q$ has no l.u.b., then the l.u.b. of $T$ is $(w, 0)$, where $w$ is the least proper successor of $u$.

Suppose that there is no greatest element of $P$. Then the l.u.b. of $T$ is (l.u.b. of $P, 0$ ). A dual argument completes the proof that $\sum_{R} S_{r}$ is a lattice [complete lattice].

We list several results in the following table. 
Property of $\sum_{R} S_{r} \quad$ Necessary and sufficient conditions on $R$ and the $S_{r}$

(1) Chain $R$ and all $S_{r}$ are chains.

(2) Ordinal $R$ and all $S_{r}$ are ordinals.

(3) Cardinal $R$ and all $S_{r}$ are cardinals.

(4) Bounded number All $S_{r}$ are numbers, $R$ is a bounded number, $S_{0}$ contains $0, S_{I}$ contains $I$.

(5) Finite number $\quad R$ and all $S_{r}$ are finite numbers.

The necessity proofs of the above results follow almost immediately from $[3,2.2]$. If $\sum_{R} S_{r}$ is a bounded number, its lower bound is $(0,0)$, and its upper bound is $(I, I)$.

The sufficiency proofs are supplied easily with the help of $[3,2.4]$.

3. Ordered products. In this section the symbol $R_{1}$ will denote the subsystem of $R$ of all $r$ such that $S_{r}$ is not a cardinal. References to $R_{1}$ are to be considered to be deleted when $R_{1}$ does not exist.

A set of necessary and sufficient conditions that an ordered product, $\prod_{R} S_{r}$, be a lattice can be given. However, the conditions are so inelegant that the statement of the conditions and the required proof is being omitted from this paper.

Theorem 2. $\prod_{R} S_{r}$ is a complete lattice if and only if the following conditions are satisfied.

(1) All $S_{r}$ are complete lattices.

(2) $R_{1}$ is a number.

(3) $R_{1}$ satisfies the ascending chain condition.

Proof. Assume that $\prod_{R} S_{r}$ is a complete lattice. Then it is a number, and $[3,4.13]$ implies that $R_{1}$ and all $S_{r}$ are numbers and that (3) holds.

Let $r$ be in $R-R_{1}$. We shall show that $S_{r}$ is a complete lattice. Suppose that $S_{r}$ contains two distinct elements, say $a$ and $b$. Let $f_{1}(r)=a, f_{2}(r)=b$, and $f_{1}(w)=f_{2}(w)$ for $w \neq r$ in $R$. Let $f=f_{1} \bigvee f_{2}$ in $\prod_{R} S_{r}$.

Now let $k(w)=f(w)$ for $w \neq r$, and let $k(r)$ be an element that does not equal $f(r)$. Then $k$ does not follow or equal $f$, but it can be checked easily that $k$ is an upper bound of $f_{1}$ and $f_{2}$. Hence $S_{r}$ must contain only one element and so be a complete lattice.

Suppose that for some $r$ in $R_{1}, S_{r}$ is not a complete lattice. By (3), we may assume that if $w>r$ in $R, S_{w}$ is a complete lattice. Suppose that $Q$ is a subsystem of $S_{r}$ with no l.u.b. Let $f_{q}(r)=q$ for each $q$ in $Q$. Let $f_{q}(w)=I$ for any $w>r$ in $R$, and let $f_{q}(w)=s_{w}$, where $s_{w}$ is a fixed element of $S_{w}$, for each remaining element $w$ in $R$, for each element $q$ in 
$Q$. Then it is clear that $\bigvee_{g \in Q} f_{g}$ does not exist. A dual argument shows that for every $r$ in $R, S_{r}$ is a complete lattice.

Now assume that conditions (1), (2), and (3) hold. (1) implies that for $r$ in $R-R_{1}, S_{r}$ contains just one element. Therefore by $[3,4.13]$, $\prod_{R} S_{r}$ is a number.

The rest of the proof and the sufficiency proof of $[3,(7)$, p. 39] are almost identical.

Let $f_{p}, p$ in $P$, be the elements of any subsystem of $\prod_{R} S_{r}$. For $r$ in $R-R_{1}$, let $f(r)=f_{p}(r)$. For $r$ a maximal element of $R_{1}$, let $f(r)$ $=\bigvee_{p \in P} f_{p}(r)$. Then for any $r$ in $R_{1}$ such that $f$ is defined over the set $E_{r}$ of all proper successors of $r$ and $f \geqq f_{p}$ over $E_{r}$ for all $p$ in $P$, define $P(r)$ to be the set of all $p$ such that $f_{p}\left(r^{\prime}\right)=f\left(r^{\prime}\right)$ for all $r^{\prime}>r$. Let $f(r)=\bigvee_{p \in P(r)} f_{p}(r)$ if $P(r)$ is not empty; let $f(r)=0$ if $P(r)$ is empty. Then $f \geqq f_{p}$ over the system consisting of $r$ and its successors. Since (3) holds, this process defines $f$ on all of $R$ by transfinite induction. As in [3, p. 39], it can be shown that $f=\bigvee_{p \in P} f_{p}$. A dual proof would show that $\prod_{R} S_{r}$ is a complete lattice.

Several other results are listed in the following table.

Property of $\prod_{R} S_{r}$

(1) Chain

(2) Ordinal

(3) Cardinal

(4) Bounded number

(5) Finite number
Necessary and sufficient conditions on $R$ and the $S_{r}$

$R_{1}$ and all $S_{r}$ are chains, $R_{1}$ satisfies the ascending chain condition.

$R_{1}$ is a finite ordinal, all $S_{r}$ are ordinals.

All $S_{r}$ are cardinals.

All $S_{r}$ are bounded numbers, $R_{1}$ is a number, $R_{1}$ satisfies the ascending chain condition.

$R_{1}$ is a number; all $S_{r}$ are finite numbers; the set of all $r$ in $R$ such that $S_{r}$ contains more than one element is finite; if $r^{\prime \prime} \geqq r^{\prime} \geqq r$ in $R$, if $r^{\prime \prime}$ and $r^{\prime} \in R_{1}$, and $S_{r}$ contains more than one element, then $r^{\prime \prime} \geqq r$.

The necessity proofs of the above results follow almost immediately from $[3,3.9],[3,3.10]$, and $[3,4.13]$. If $\prod_{R} S_{r}$ is a bounded number and $f=I$, then $f(r)=I$ for all $r$ in $R$; if $f=0$, then $f(r)=0$ for all $r$ in $R$.

The sufficiency proofs of these results are supplied easily with the help of $[3,4.13]$.

By letting $R$ be the number consisting of the integers 1 and 2 ordered by magnitude, we could obtain as corollaries of our results stated so far several easily proved or known results (see, for example, [1] and [2]) concerning the ordinal sum and product of two ordered 
systems. Similarly, results for the cardinal sum and product of ordered systems and some of the results in [3] concerning the ordinal power of an ordered system can be obtained.

We shall state just one corollary of Theorem 1 giving necessary and sufficient conditions for the ordinal product of two ordered systems to be a lattice. Our excuse for stating this corollary is that the conditions as given in $[2$, p. 25, Ex. 2] are not necessary, as is stated there, but are only sufficient.

COROLlary. $R \circ S$ is a lattice if and only if the following conditions are satisfied.

(1) $R$ is a lattice, and $S$ is a number.

(2) If $R$ is not a chain, then $S$ is bounded.

(3) If there are two elements in $S$ without a l.u.b., then they have no upper bound, $S$ contains 0 , and every element in $R$ has a least proper successor.

(4) If there are two elements in $S$ without a g.l.b., then they have no lower bound, $S$ contains $I$, and every element in $R$ has a greatest proper predecessor.

\section{Bibliography}

1. G. Birkhoff, Generalized arithmetic, Duke Math. J. vol. 9 (1942) pp. 283-302.

2. - Lattice theory, Amer. Math. Soc. Colloquium Publications, vol. 25, rev. ed., New York, 1948.

3. M. M. Day, Arithmetic of ordered systems, Trans. Amer. Math. Soc. vol. 58 (1945) pp. 1-43.

Swarthmore College 